\title{
EUVL: the natural evolution of optical microlithography
}

\section{Bernd Geh}

Bernd Geh, "EUVL: the natural evolution of optical microlithography," Proc. SPIE 10957, Extreme Ultraviolet (EUV) Lithography X, 1095705 (18 October 2019); doi: $10.1117 / 12.2515791$

SPIE. Event: SPIE Advanced Lithography, 2019, San Jose, California, United States 

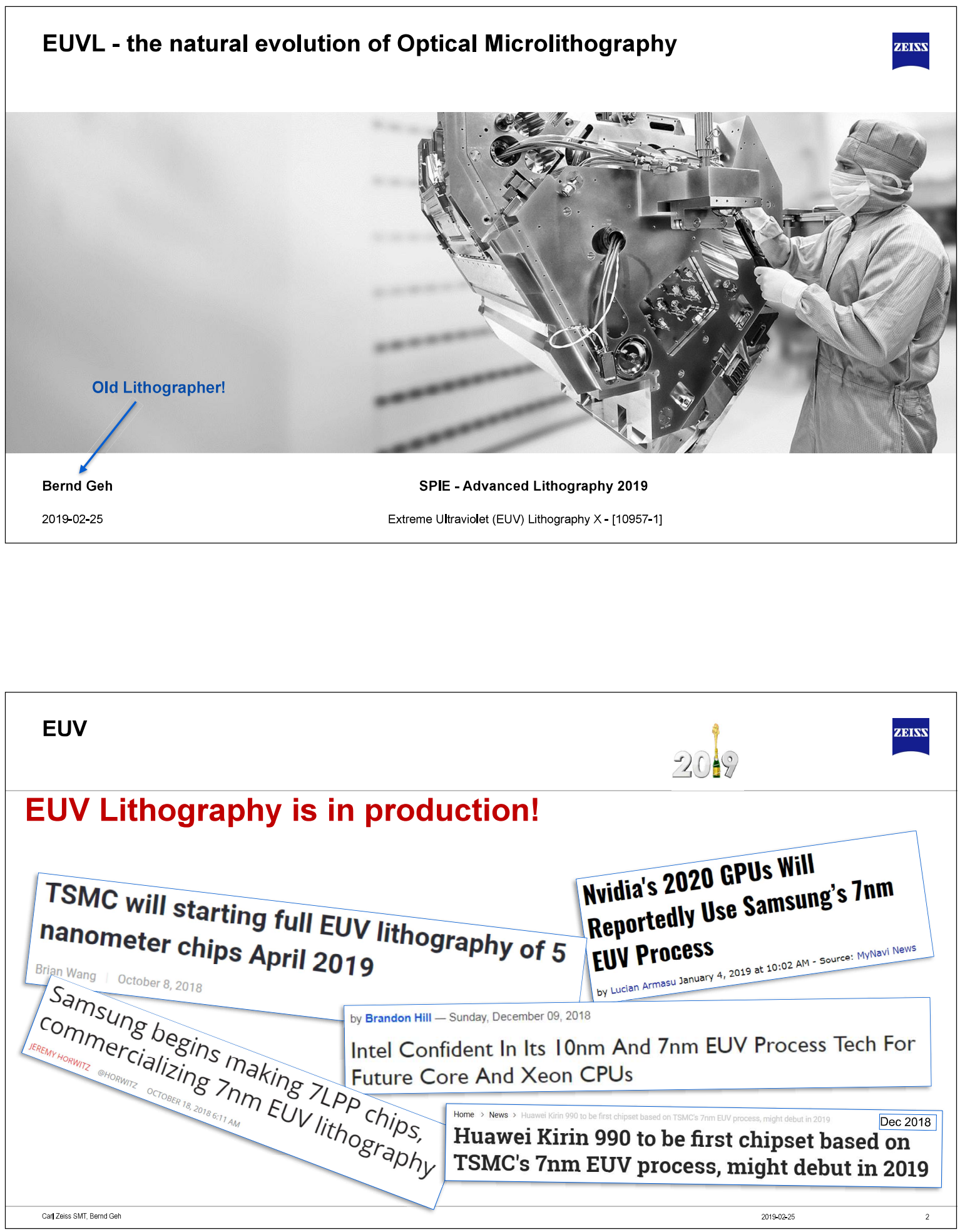

Extreme Ultraviolet (EUV) Lithography X, edited by Kenneth A. Goldberg, Proc. of SPIE Vol. 10957, 1095705 - @ 2019 SPIE · CCC code: 0277-786X/19/\$18 · doi: 10.1117/12.2515791 


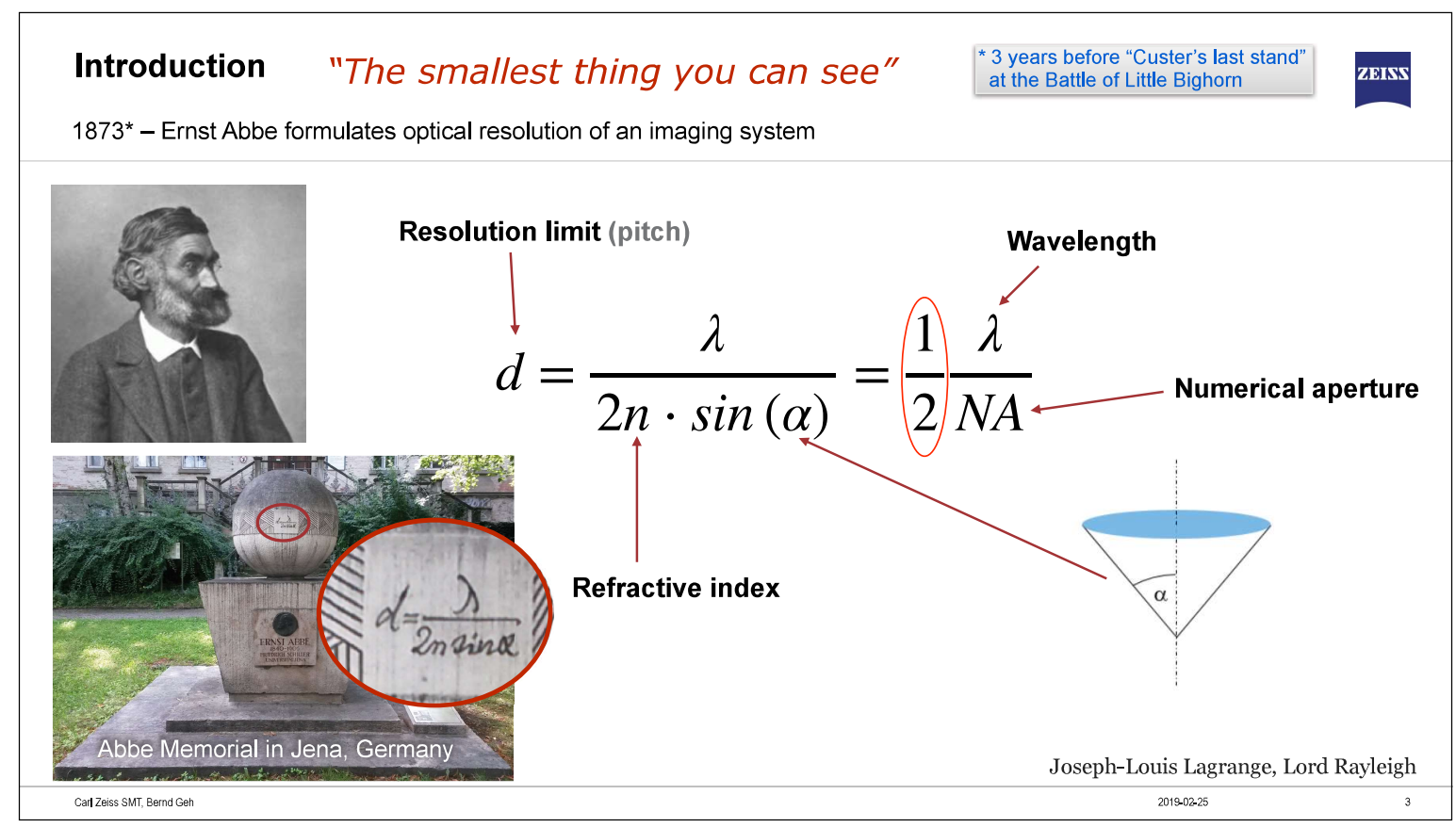

Introduction

"The smallest thing you can make"

Burn J. Lin, "Where Is The Lost Resolution?," Proc. SPIE 0633, Optical Microlithography V, (20 August 1986);

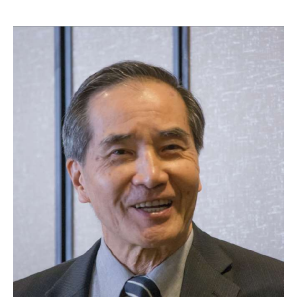

Smallest feature size (Half pitch)

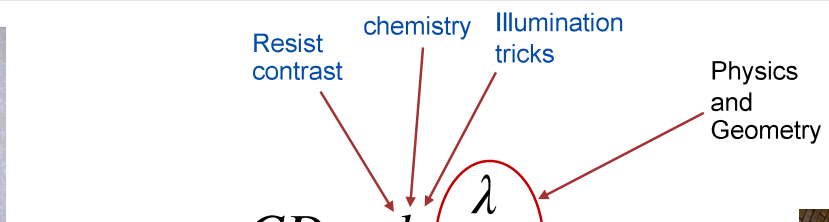

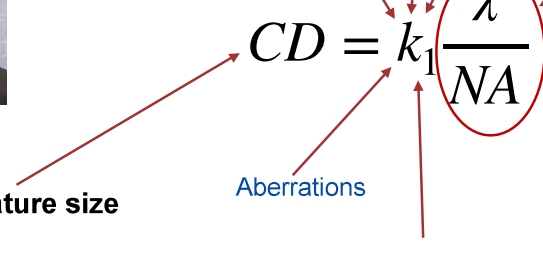

Mask tricks (OPC, RET, PSM)

$\mathbf{k}_{1}$ became a simple measure on how well we push the limits of physics...

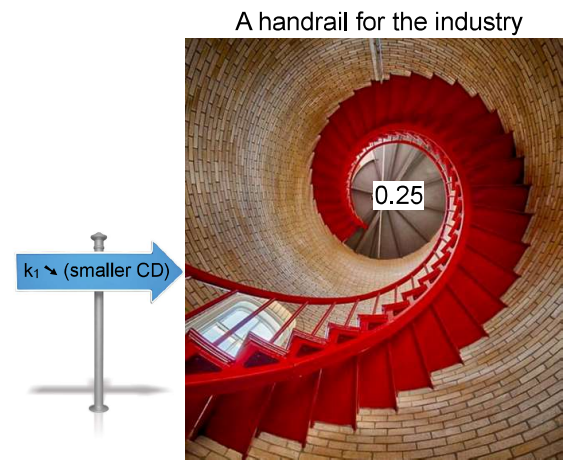




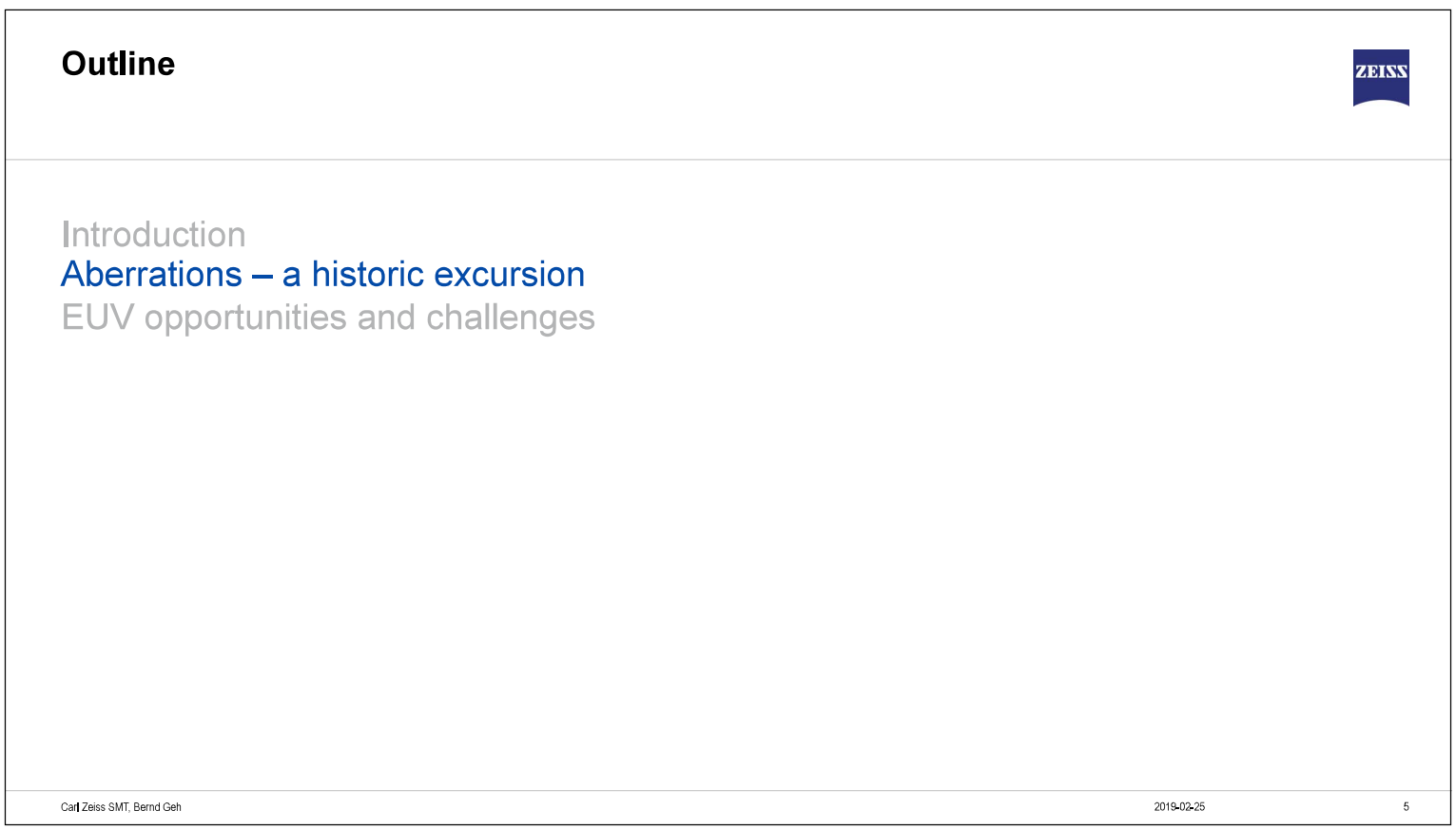

Aberrations - A historical overview

Lens aberrations in comparison

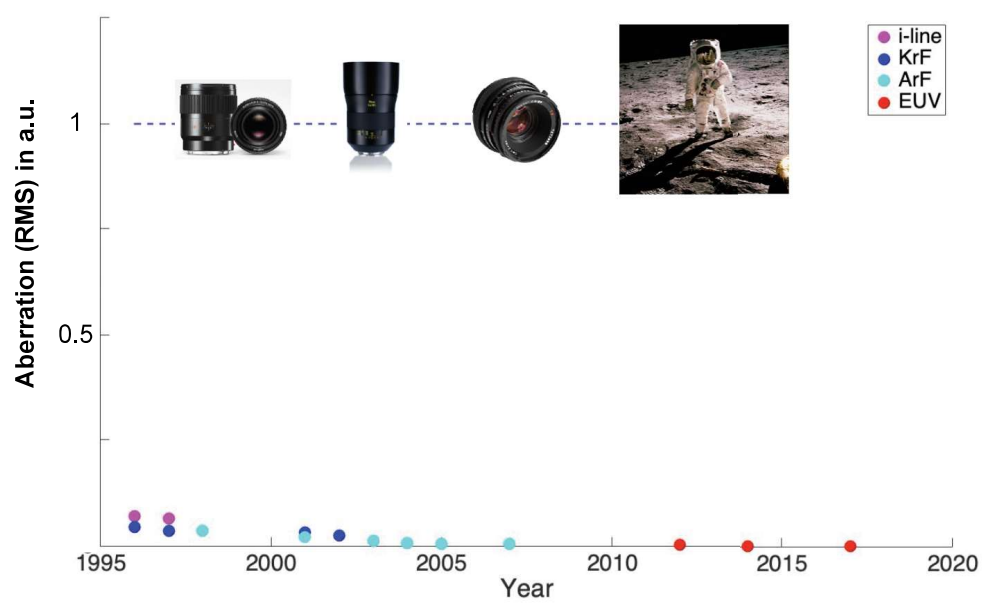

Lithography lenses have always been orders of magnitudes more perfect than even State of the Art Camera Lenses

Carl Zeiss SMT, Bernd Geh

2019-02-25 


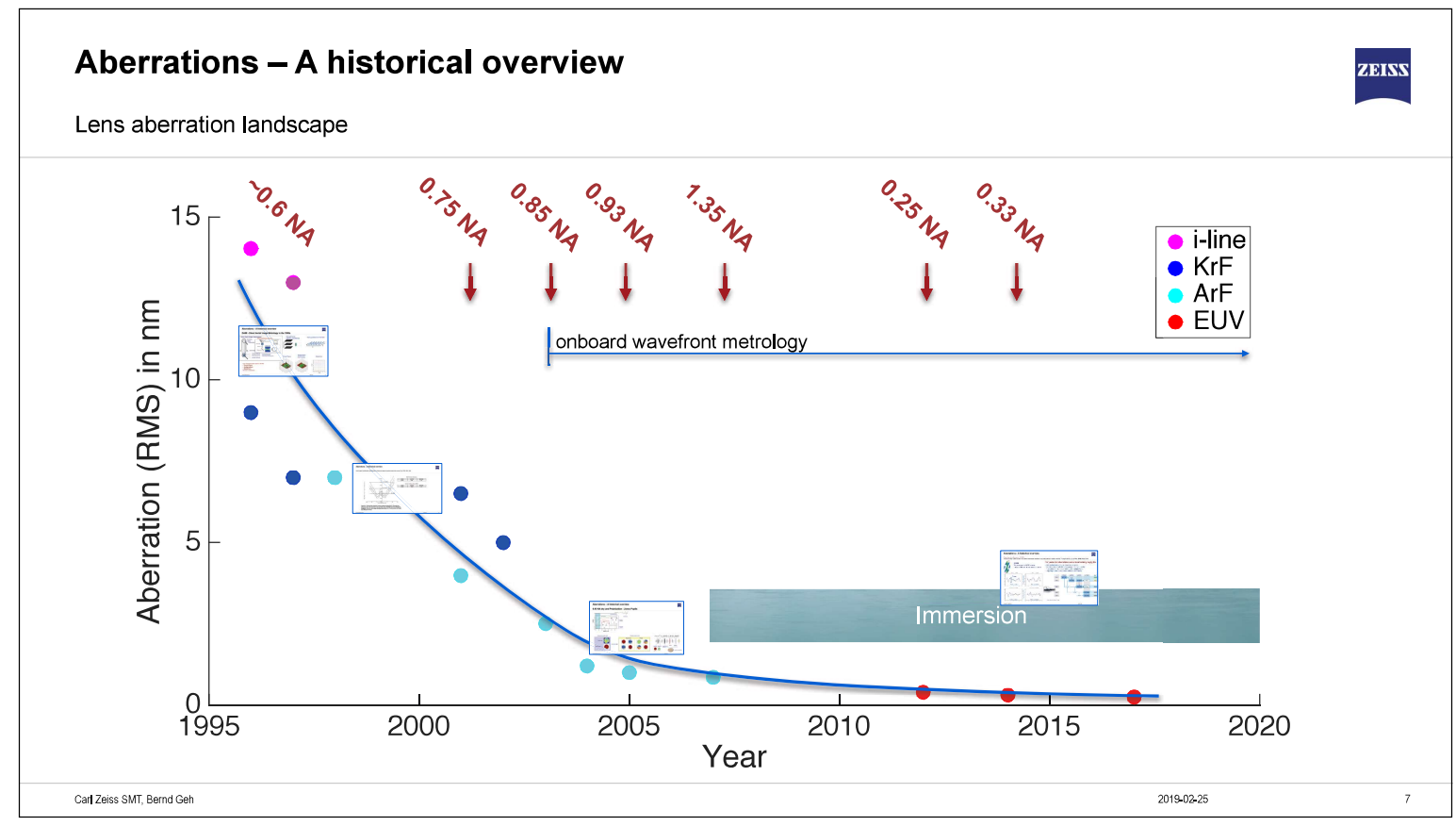

Aberrations - A historical overview

mid 90s

1995: Lenses were characterized by the location of the aerial image $x, y$ and $z$

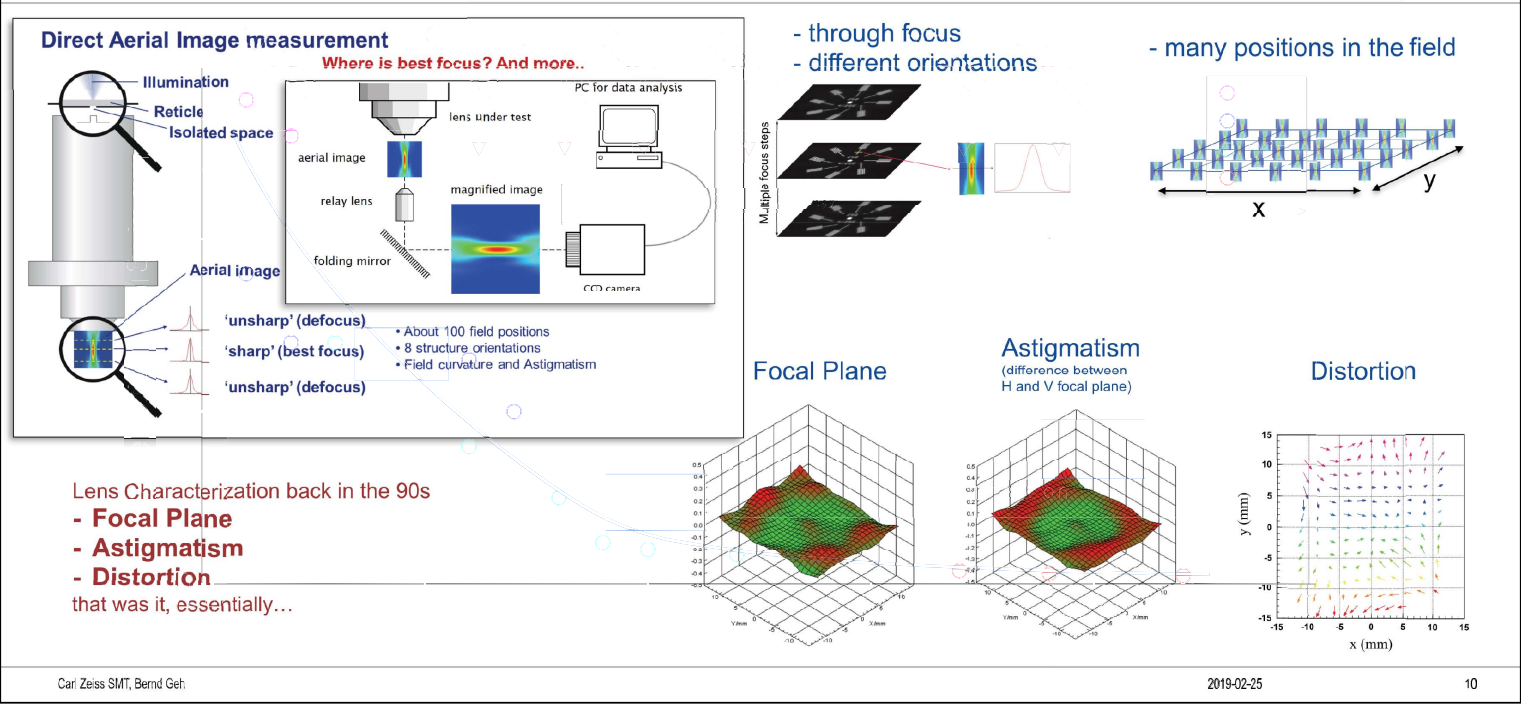

Proc. of SPIE Vol. 10957 1095705-4 


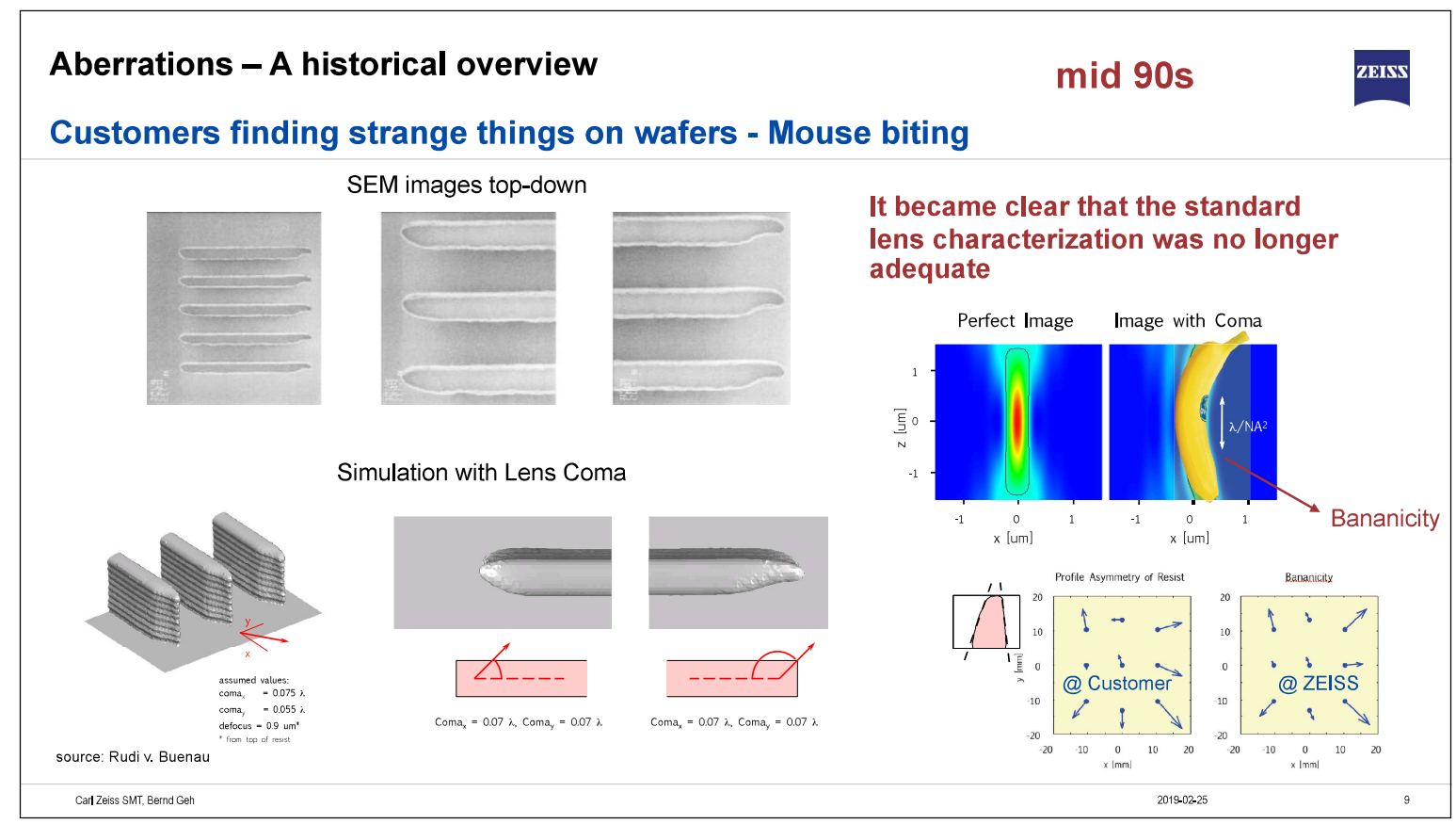

Aberrations - A historical overview

Push towards actinic through-the-lens aberration measurements

mid 90s Both internal and also external
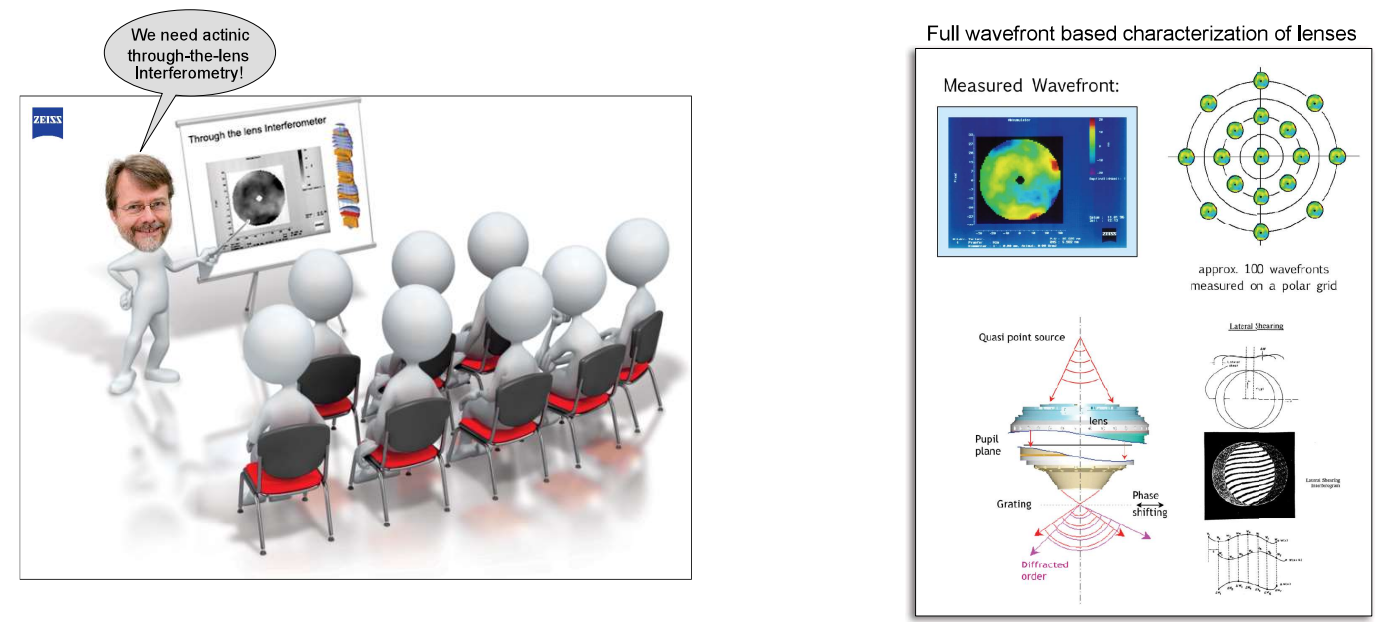


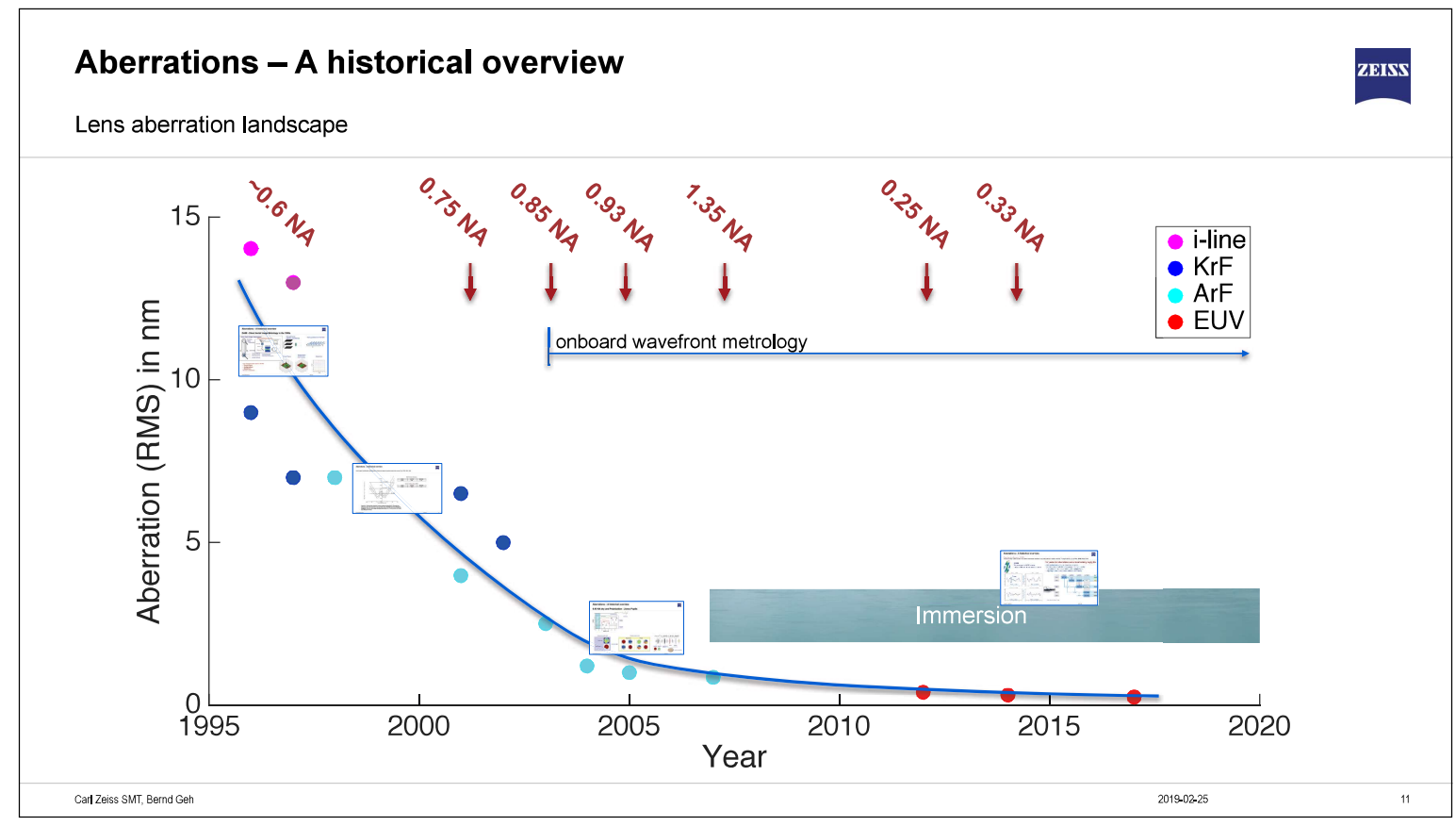

Aberrations - A historical overview

1999

Chris Progler, Scott Bukofsky, Donaid Wheeler, Method to budget and optimize total device overlay, Proc. SPIE. 3679, 1999

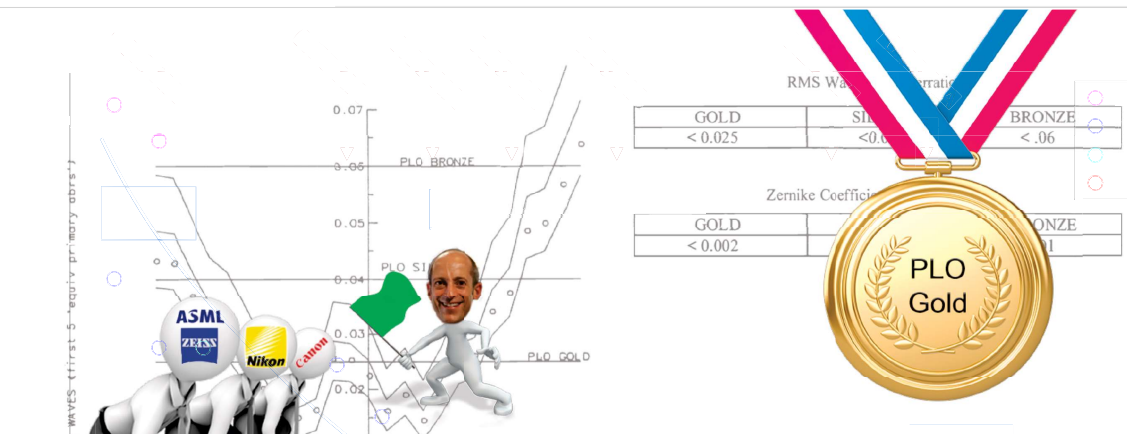

\section{Progler Lens Olympic}

Figure \#10. The folal RSS of the first 5 EPAA's plotted in Figures \#8 \& 9. The points are

measured values. The central line is the most likely value of the RMS wave front aberrations,

bounded by 1 std. dev. error ranges including measurement error. The horozontals are Progle

Lens Olympic levels [2] 


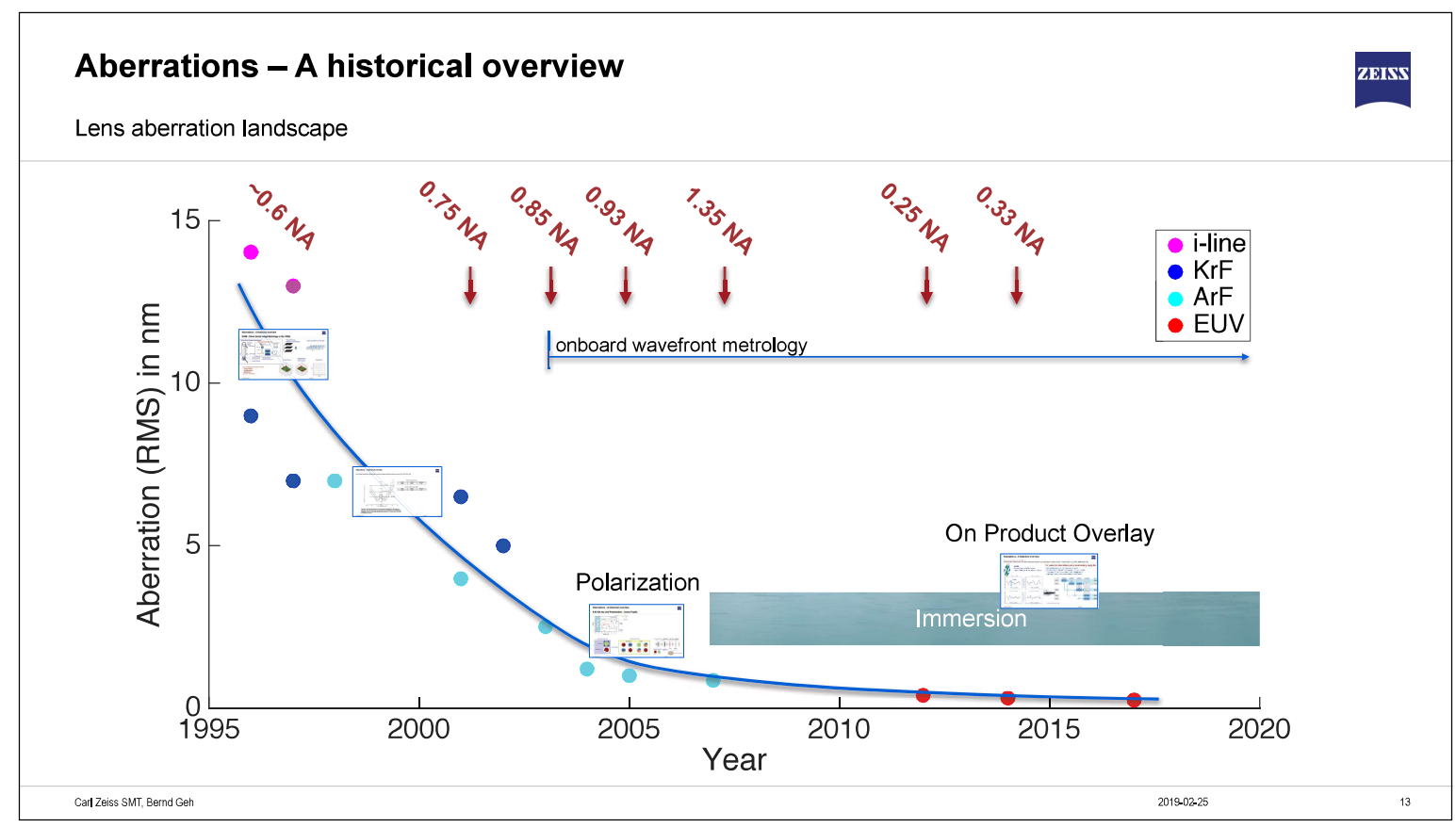

\section{Outline}

Introduction

Aberrations - a historic excursion

EUV opportunities and challenges 


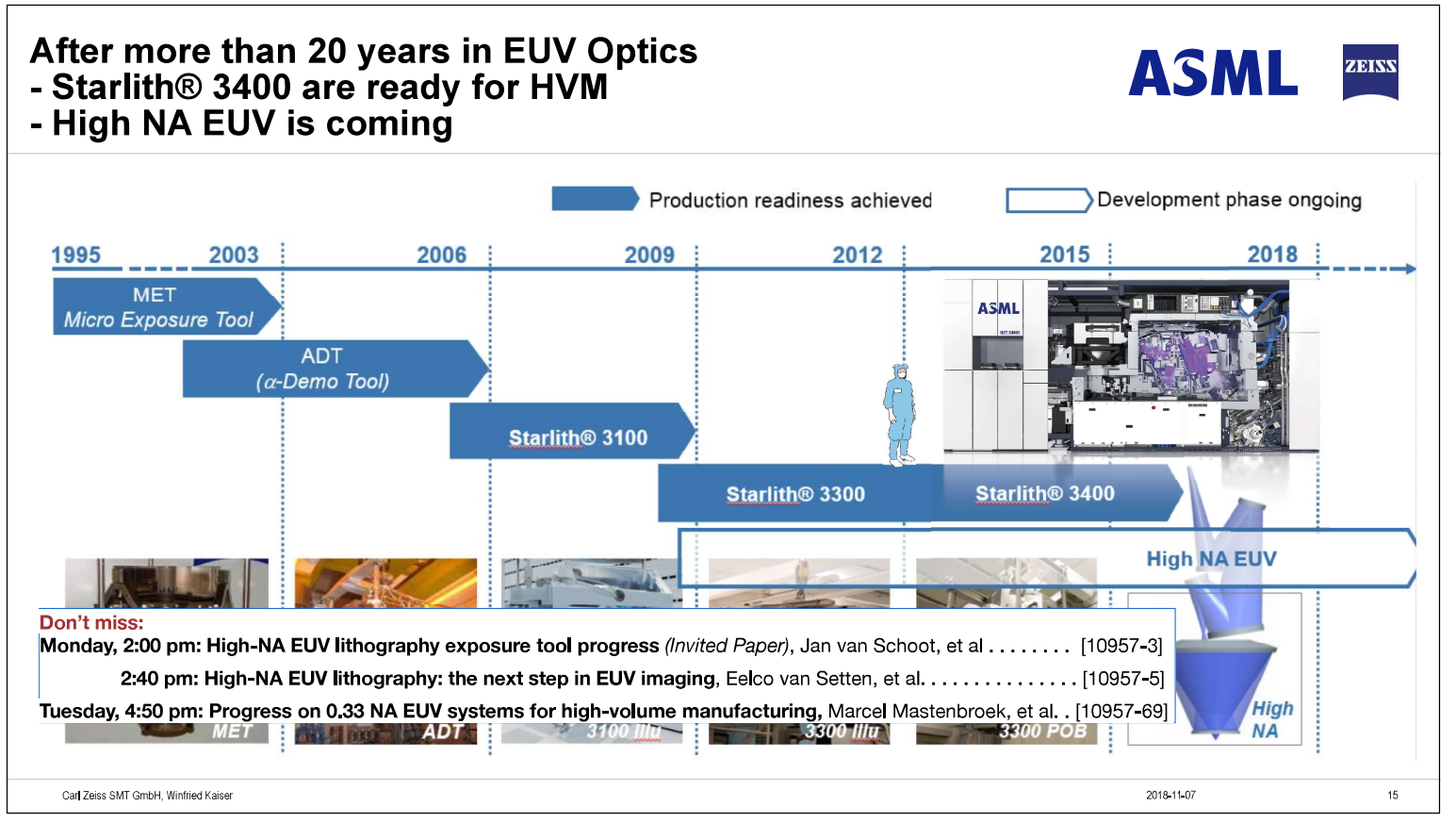

\section{High NA EUV}

Construction status ZEISS Oberkochen in Nov 2018

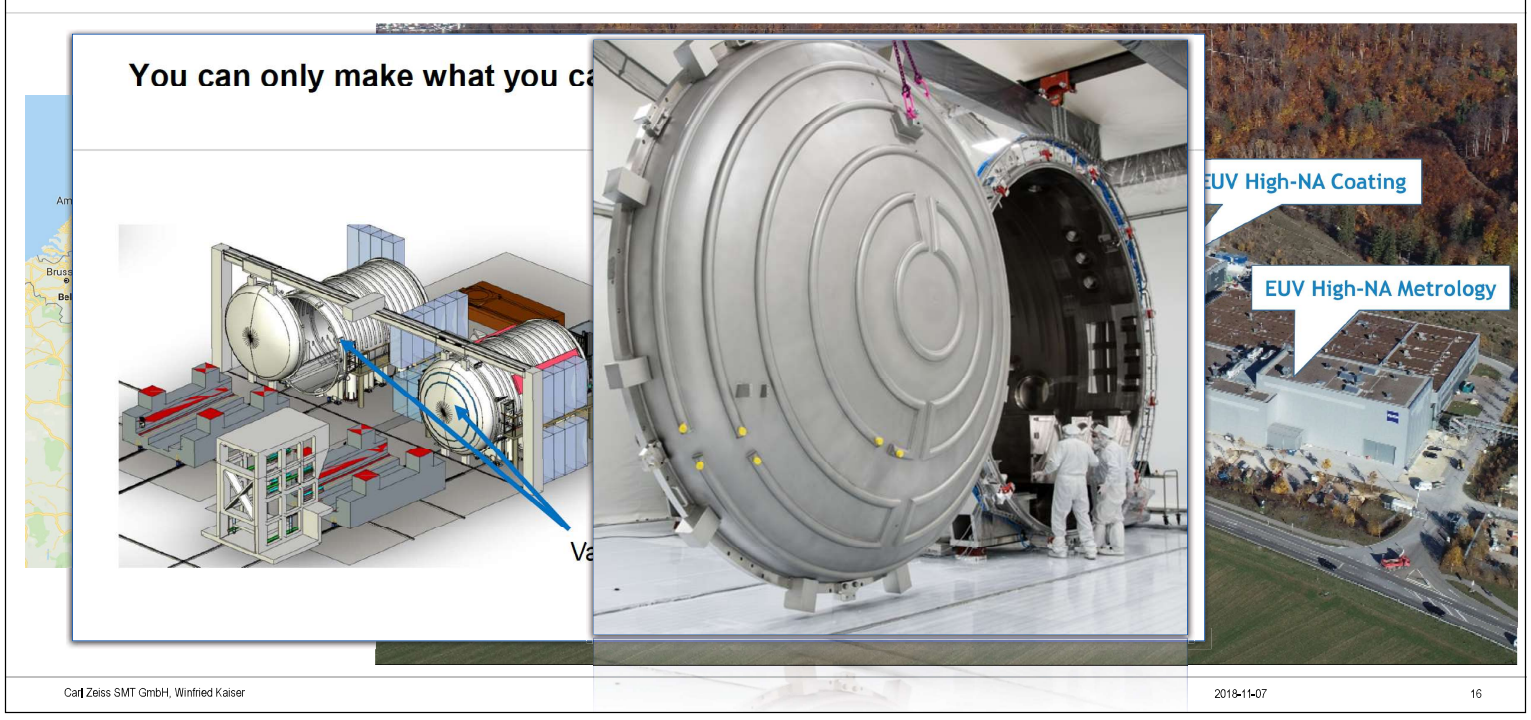




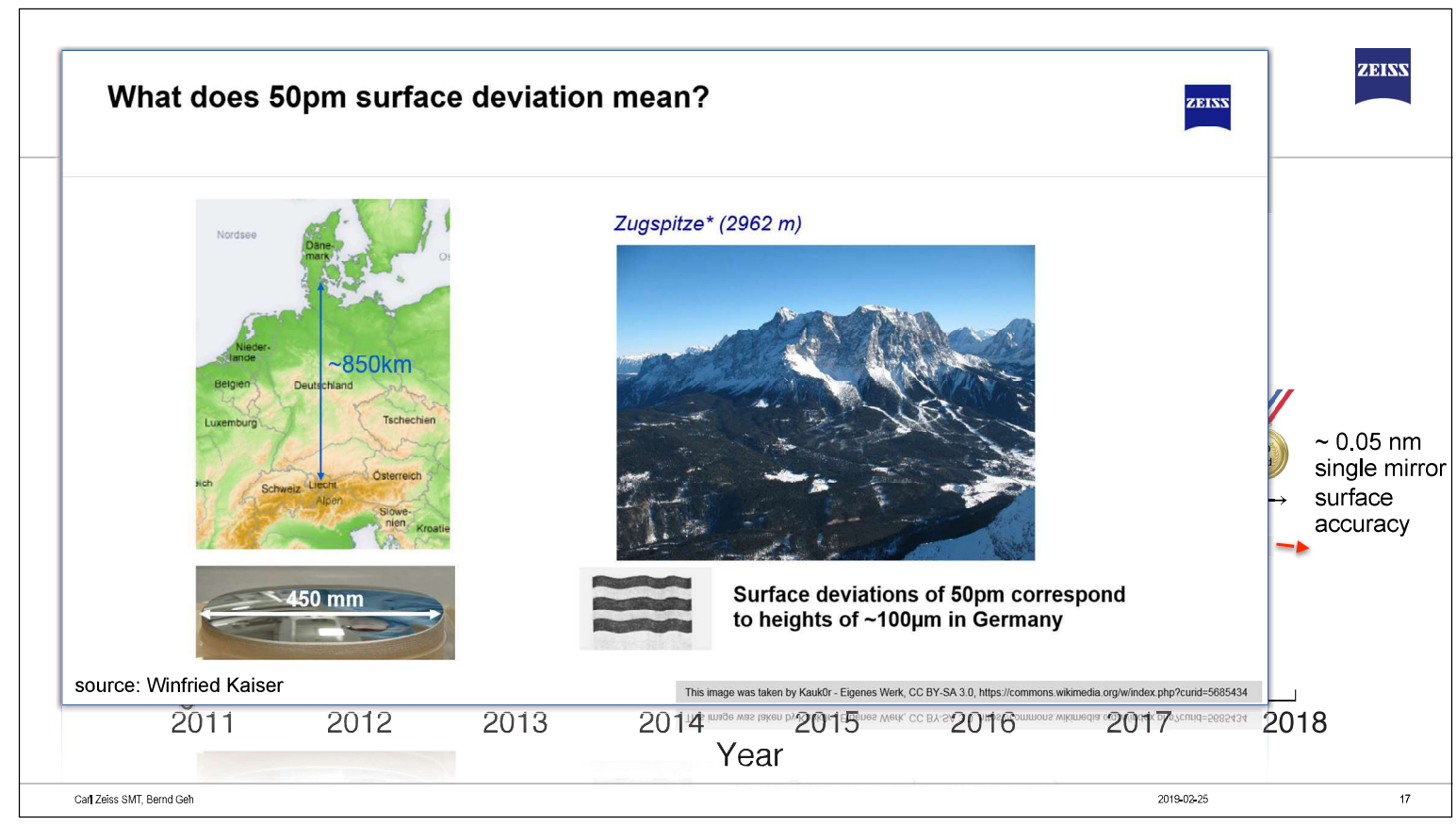

\section{ZEISS EUV infrastructure support}

ZEISS ForTune TM Process EUV-ArFi Overlay matching improvement

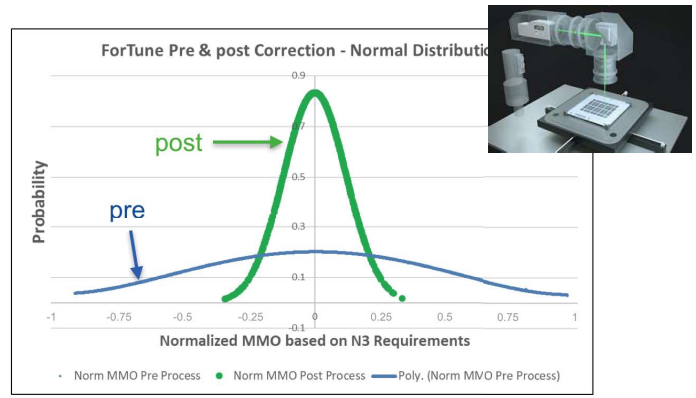

$80 \%$ NXE-NXT matching improvement

Don't miss: Poster session, Wednesday, Enhanced wafer overlay residuals control; deep sub-nanometer at sub-millimeter lateral resolution,

Avi Cohen, Philippe Leray, et al., Carl Zeiss SMS Ltd. and IMEC .........[10959-91]

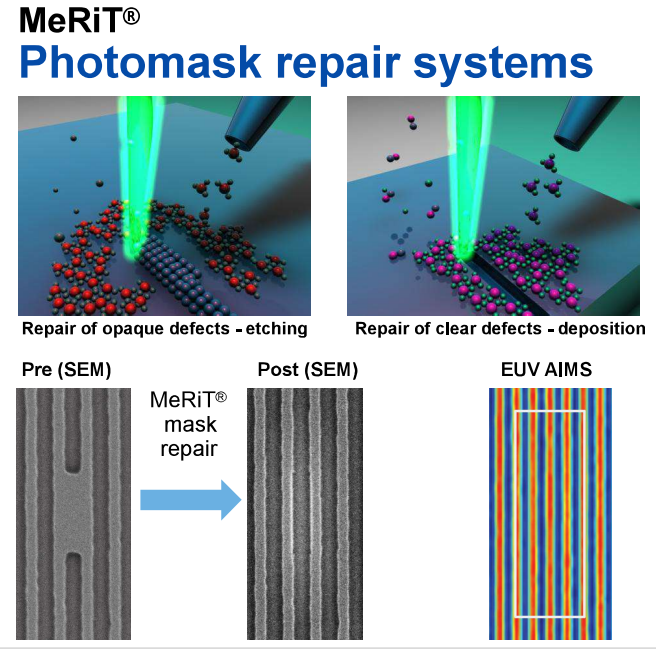

2019-02-25 


\section{ZEISS AIMSTM EUV for mask 3D effects qualification \\ "Seeing the mask like the scanner does" \\ Mask Metrology zarss}

Full qualification of mask 3D effects, and their dependence on process parameters

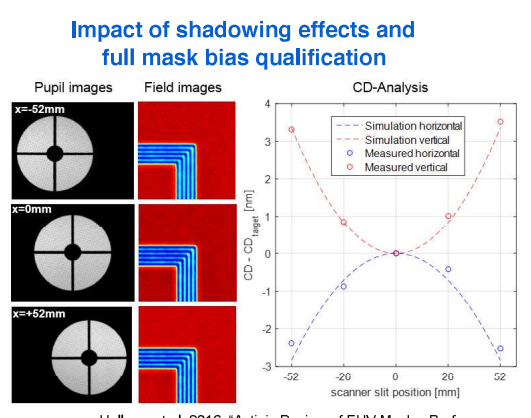

Hellweg et al. 2016, "Actinic Review of EUV Masks: Performance
Data and Status of the AlMSTM EUV Systen
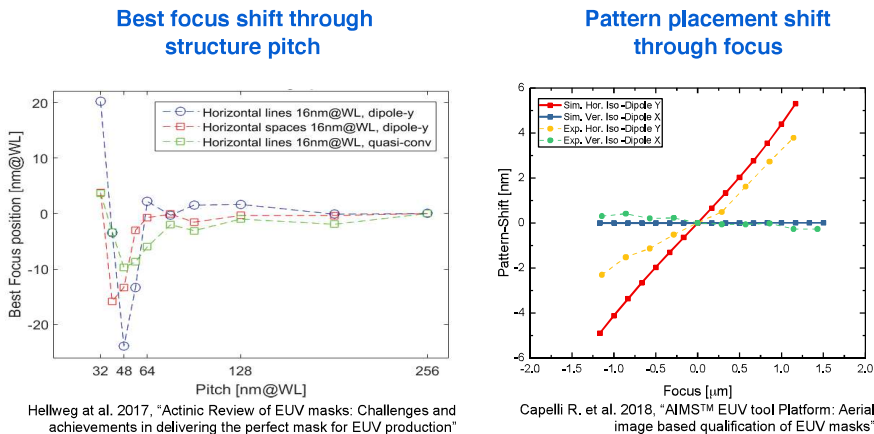

image based qualification of EUV masks'

Don't miss: Wednesday 11:30 am: Actinic metrology platform for defect review and mask qualification: flexibility and performance,

Renzo Capelli, Martin Dietzel, Dirk Hellweg, Grizelda Kersteen, Conrad Wolke, Carl Zeiss SMT GmbH (Germany)

$[10957-66]$

\section{EUV - Alternative Absorber Materials}

Optimizing Absorber properties to reduce 3D effects and shadowing

\section{n\&k REGIONS FOR IMAGING IMPROVEMENT vs. REFERENCE TaBN}

Dependent on imaging metric

source: Vicky Philipsen, EUV Photomask, 2018
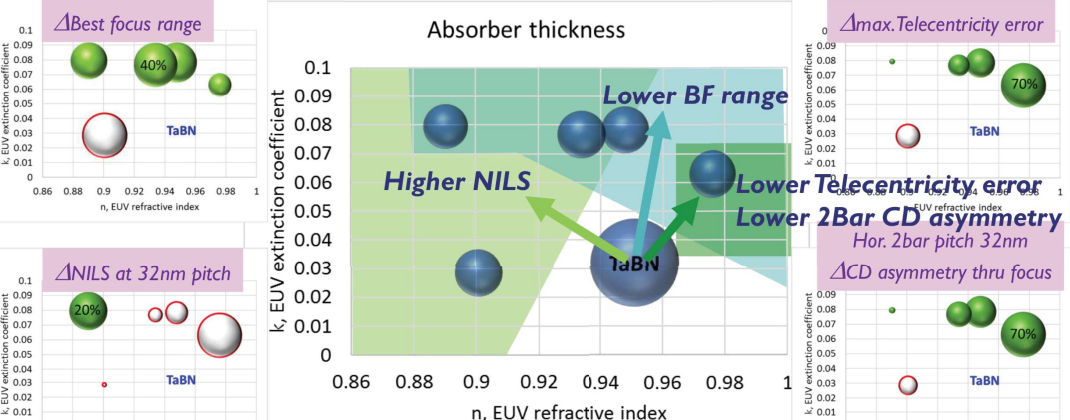

Regions for imaging improvement compared to reference TaBN identified

Weight of optimization metric determines superior $n \& k$ region

\section{เйec}

Don't miss: Wednesday 4:10 pm: Experimental Investigation of a high-k reticle absorber system for EUV lithography, Jo Finders, et al. ..... [10957-37] 


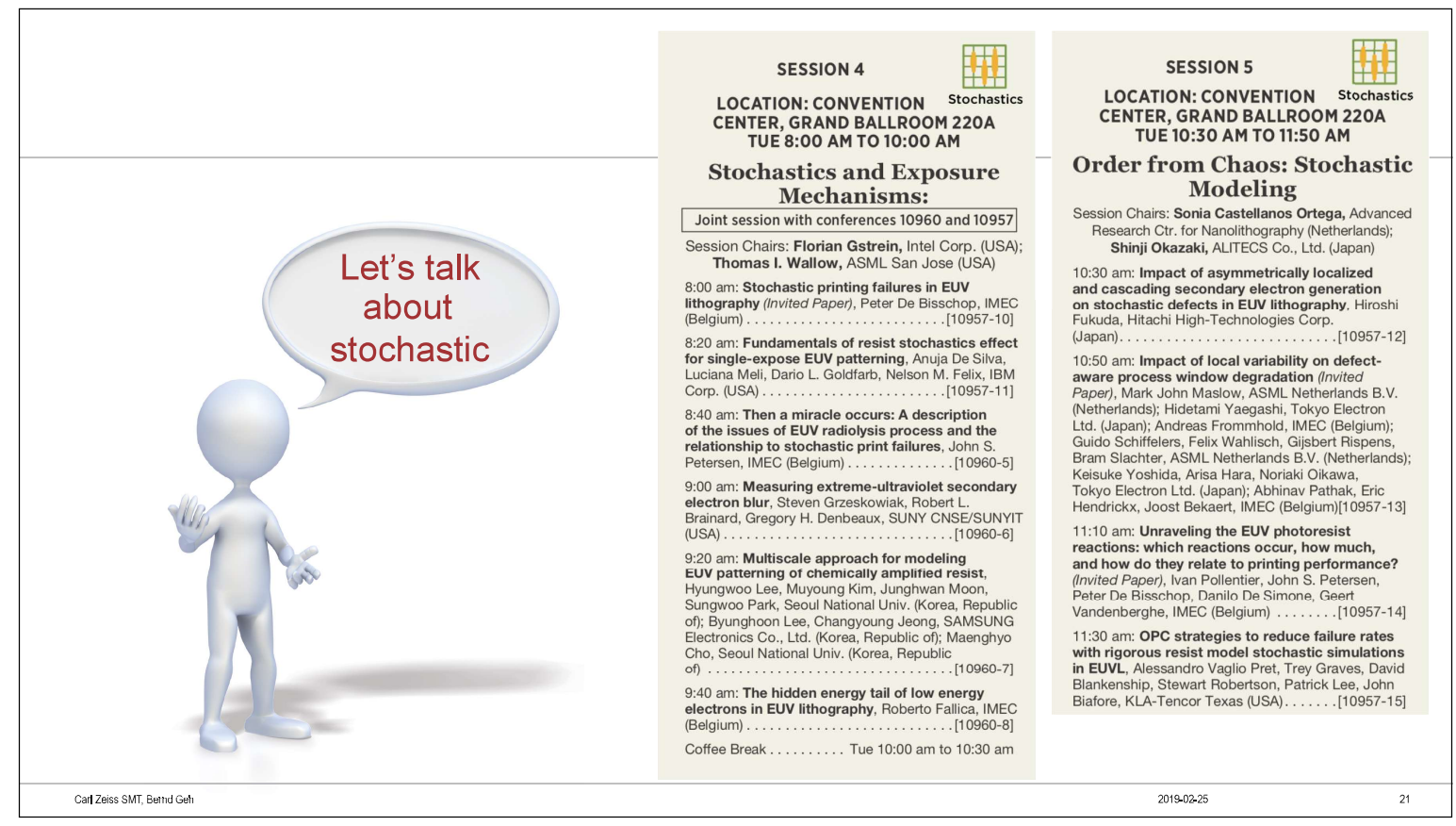

\section{EUVL - Photon statistics}

Wrapping the head around LER and LCDU

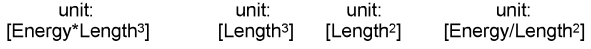

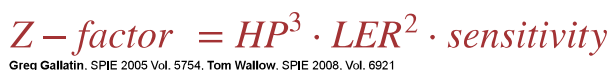

$$
\begin{aligned}
& \text { unit: unit: unit: } \\
& \underset{\text { Steve Hansen, JMs } 2018 \text { Vol. } 17}{L C D}=a \cdot I L S^{b}
\end{aligned}
$$

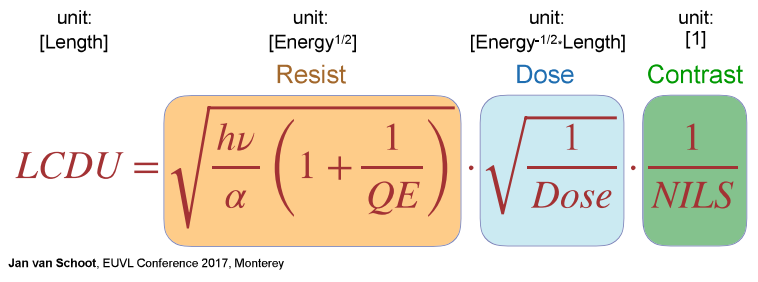




\section{Photon statistics - revisited}

You need a number of photons to calculate a fluctuation

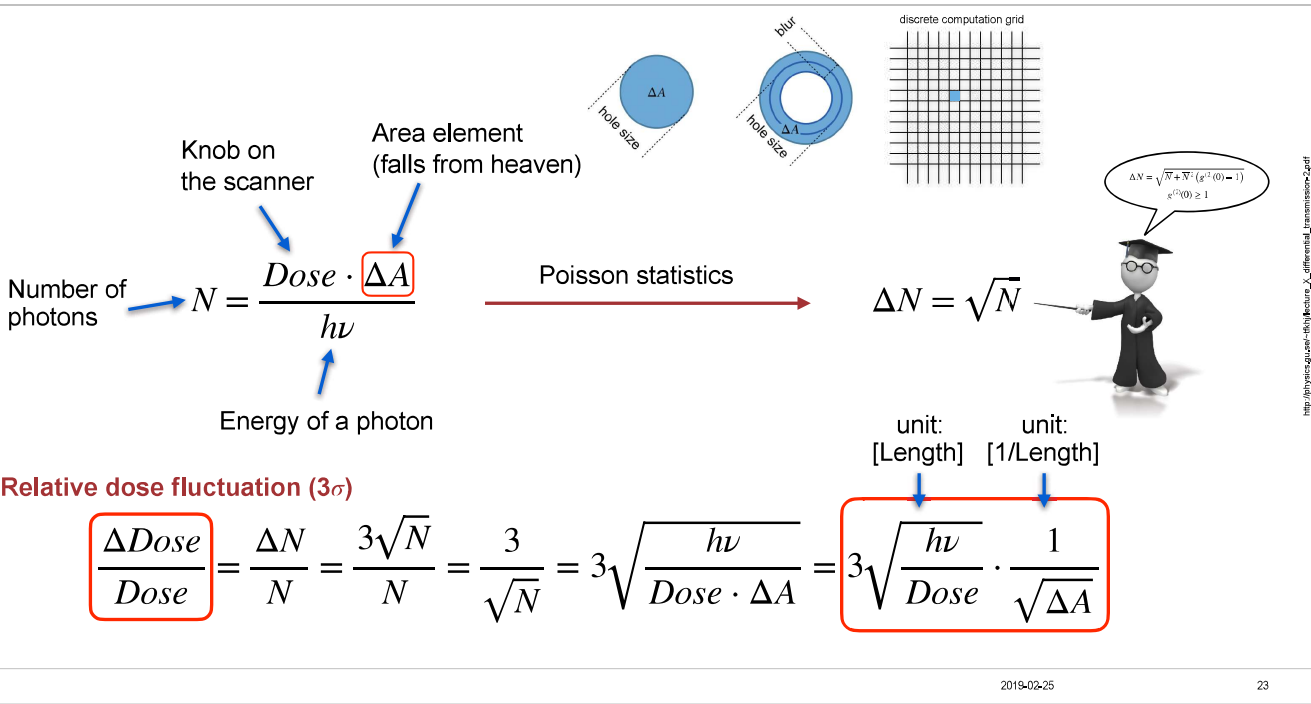

\section{Photon statistics - revisited}

How Dose fluctuation turns into edge placement

$D \cdot I(x)=D_{0} \quad \Rightarrow \quad \Delta D \cdot I(x)+D \cdot I^{\prime}(x) \Delta x=0 \quad \Rightarrow \quad-\frac{\Delta D}{D}=\frac{I^{\prime}(x)}{I(x)} \Delta x=I L S \cdot \Delta x$

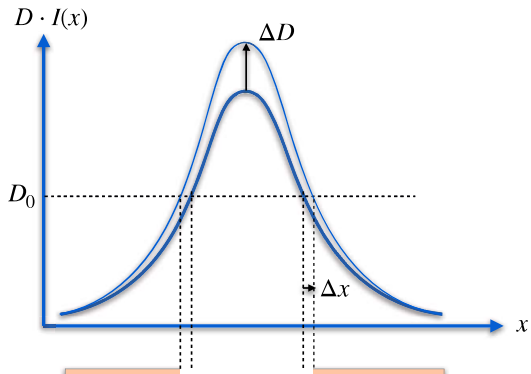

unit:
[Length]

$\begin{array}{cc}\text { unit: } & \text { unit: } \\ \text { [Length } & \text { unit: } \\ \text { [1/Length] }\end{array}$

$\Delta x=\frac{1}{I L S} \frac{\Delta D}{D}=\frac{1}{I L S} \frac{\Delta N}{N}=\frac{3}{I L S} \cdot \sqrt{\frac{h \nu}{D o s e}} \cdot \frac{1}{\sqrt{(\Delta A}}$

Image

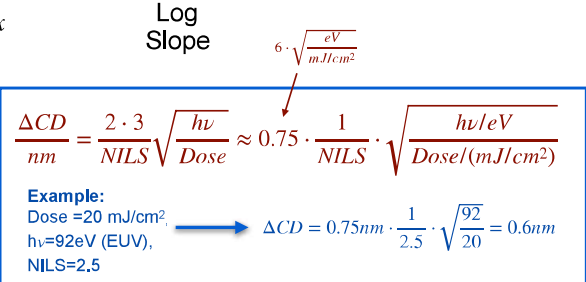

Still have to make a choice as to what $\Delta A$ is

Can just as well choose $\mathrm{CD}^{2}$ and multiply it to ILS to make it NILS

$\sqrt{\Delta A} \cdot I L S=C D \cdot I L S=N I L S$ 
Photon statistics - revisited

A more detailed "sing along" version - FOR HANDOUT

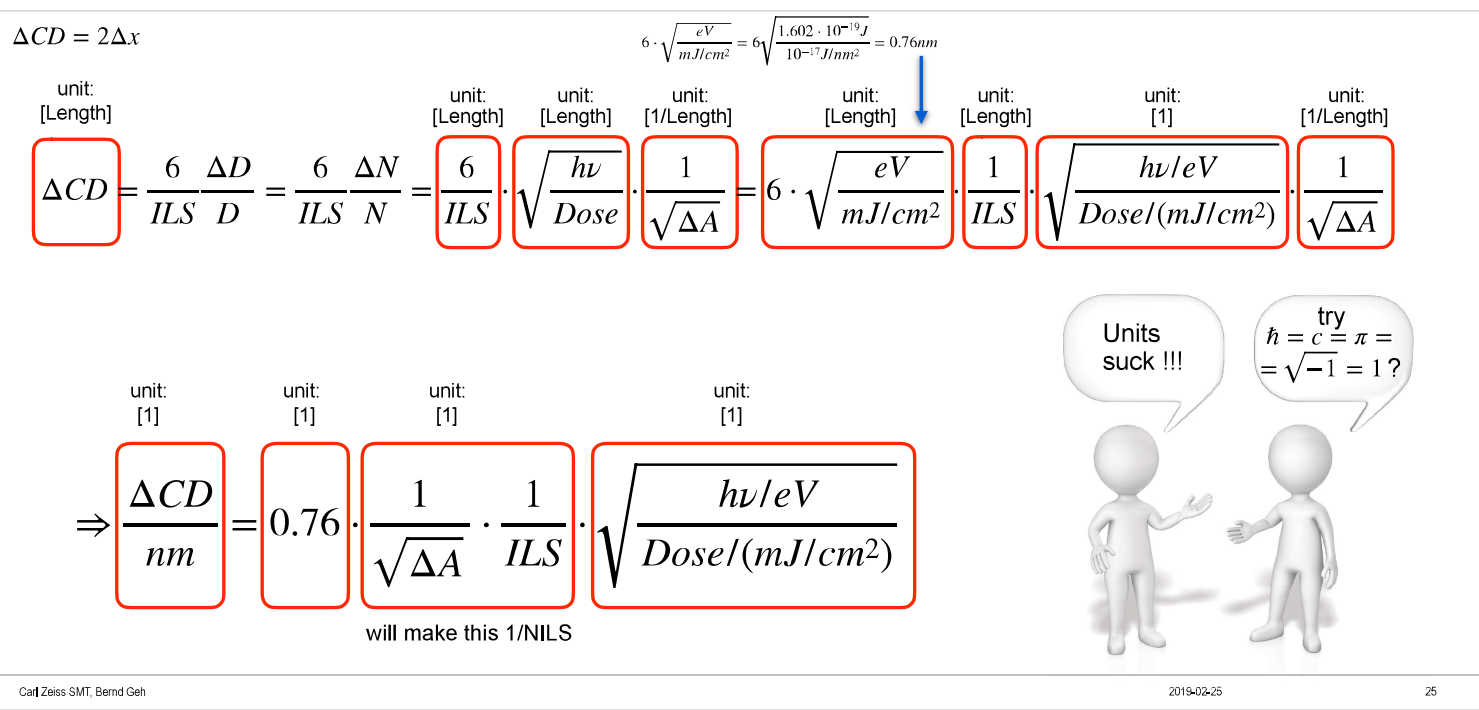

\section{Photon statistics - revisited}

How Dose fluctuation turns into edge placement

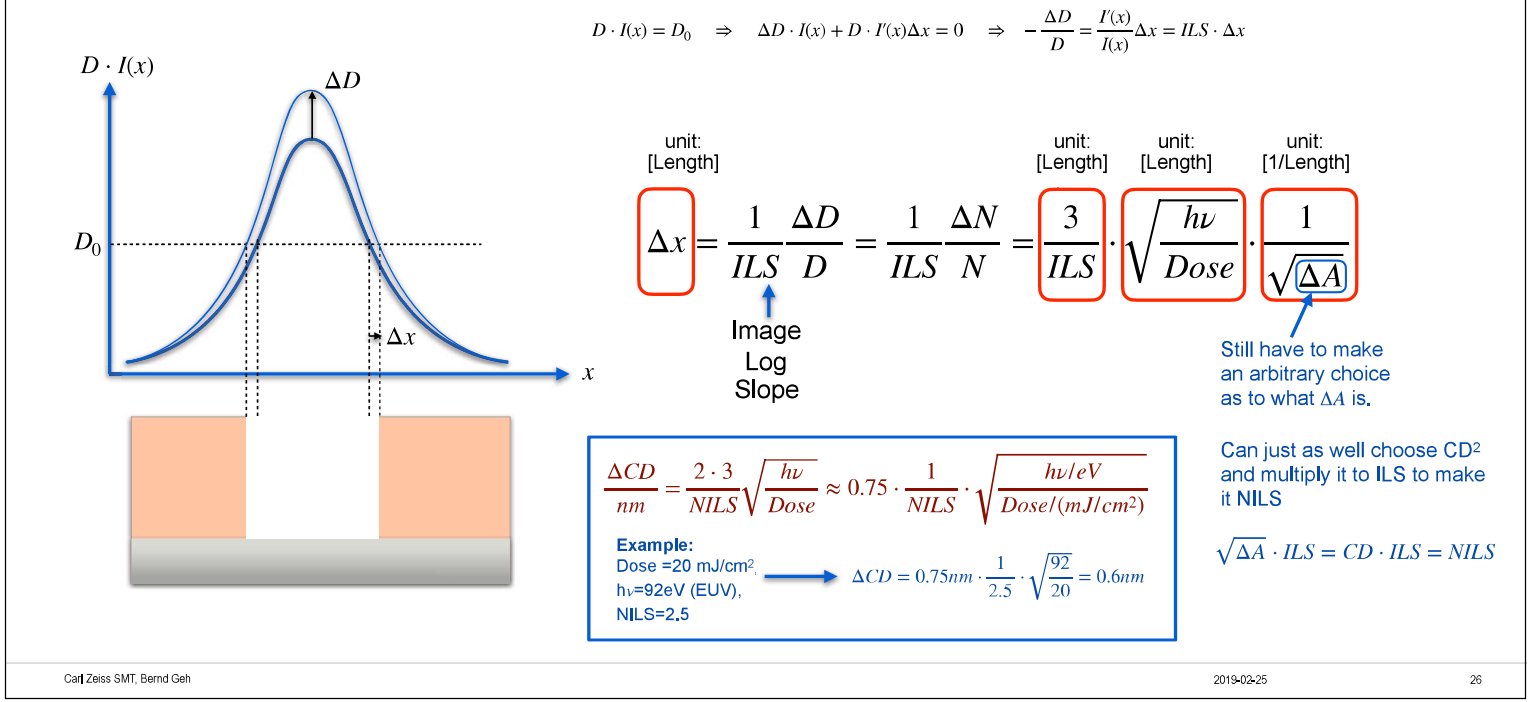




\section{Photon statistics - revisited}

How Dose fluctuation turns into edge placement

$$
\frac{L C D U}{n m} \approx 0.75 \cdot \frac{1}{N I L S} \cdot \sqrt{\frac{h \nu / e V}{D o s e /\left(m J / \mathrm{cm}^{2}\right)}}
$$

\section{EUV - Stochastics}

Following Burn Lin's footsteps

$$
C D=k_{1} \frac{\lambda}{N A} \quad \text { DoF }=k_{2} \frac{\lambda}{N A^{2}} \quad \text { DoF }=k_{3} \frac{\lambda}{\sin (\theta / 2)^{2}}
$$

$$
\frac{L C D U}{n m}=k_{4} \cdot \frac{1}{N I L S} \cdot \sqrt{\frac{h \nu / e V}{\operatorname{Dose} /\left(\mathrm{mJ} / \mathrm{cm}^{2}\right)}}
$$


EUV - Stochastics

Following Burn Lin's footsteps

$$
C D=k_{1} \frac{\lambda}{N A} \quad D O F=k_{2} \frac{\lambda}{N A^{2}} \quad D o F=k_{3} \frac{\lambda}{\sin (\theta / 2)^{2}}
$$

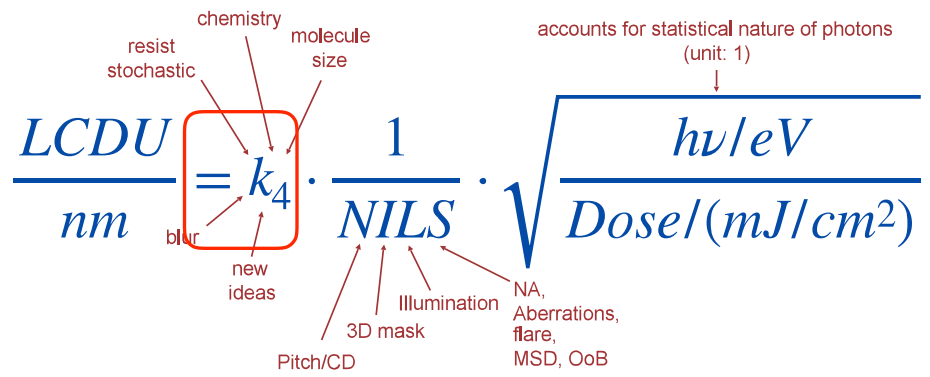

Today at NILS $=2.5, \mathrm{~h} v=92 \mathrm{eV}$ and reported LCDU values around $3-4 \mathrm{~nm} \Rightarrow \mathrm{k}_{4}$ is between 4 and 8 .

The photon field itself would have $k_{4}$ of 0.75

$$
\begin{aligned}
& 2 \text { beam imaging (small features): } \\
& N I L S=2 \ldots \pi \Rightarrow \frac{1}{N I L S}=0.5 \ldots 0.32
\end{aligned}
$$

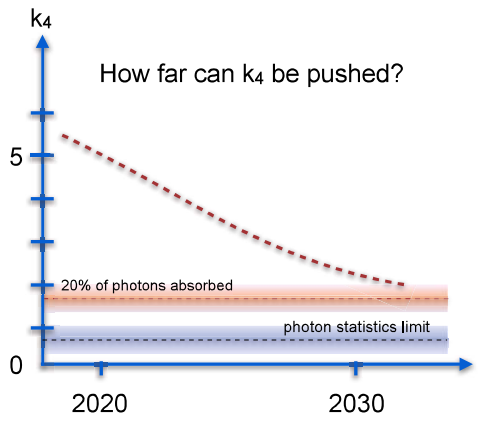

\section{$k_{4}$ - landscape 2018}
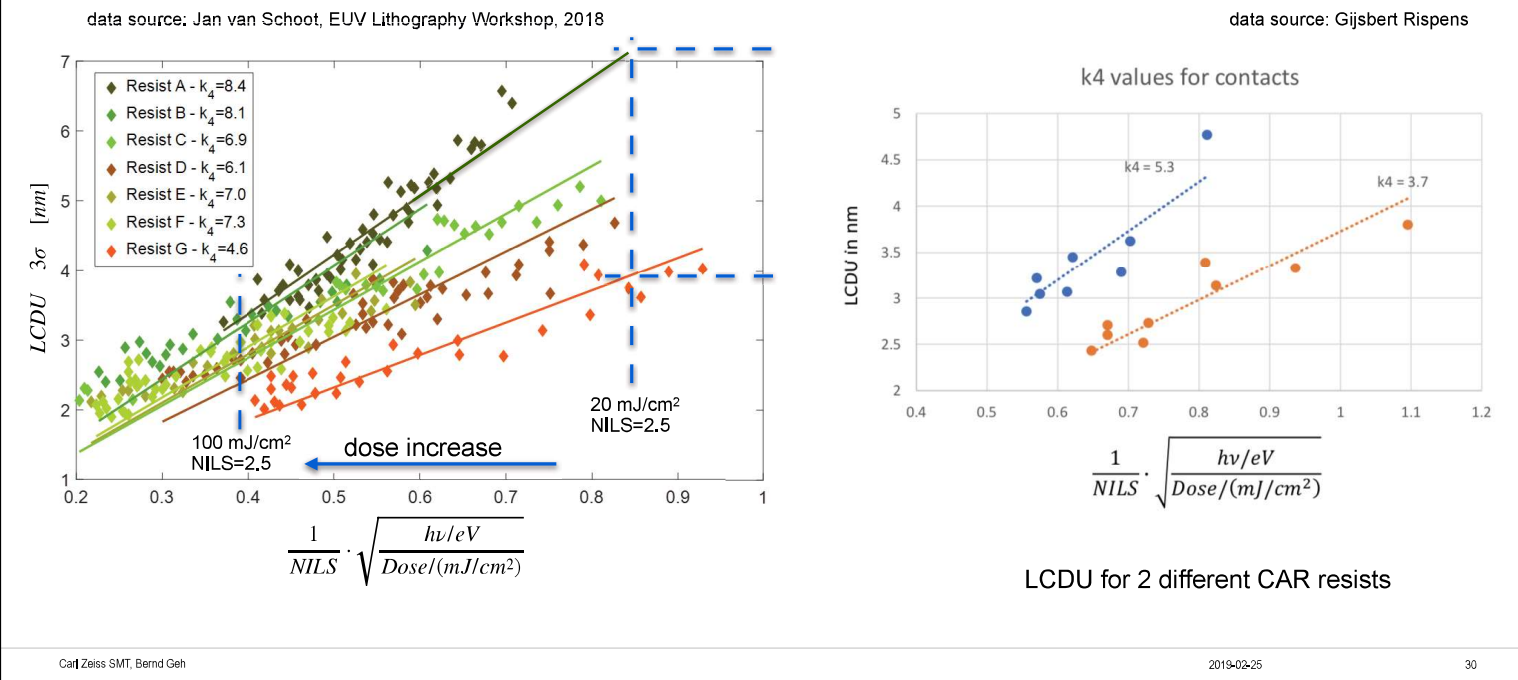

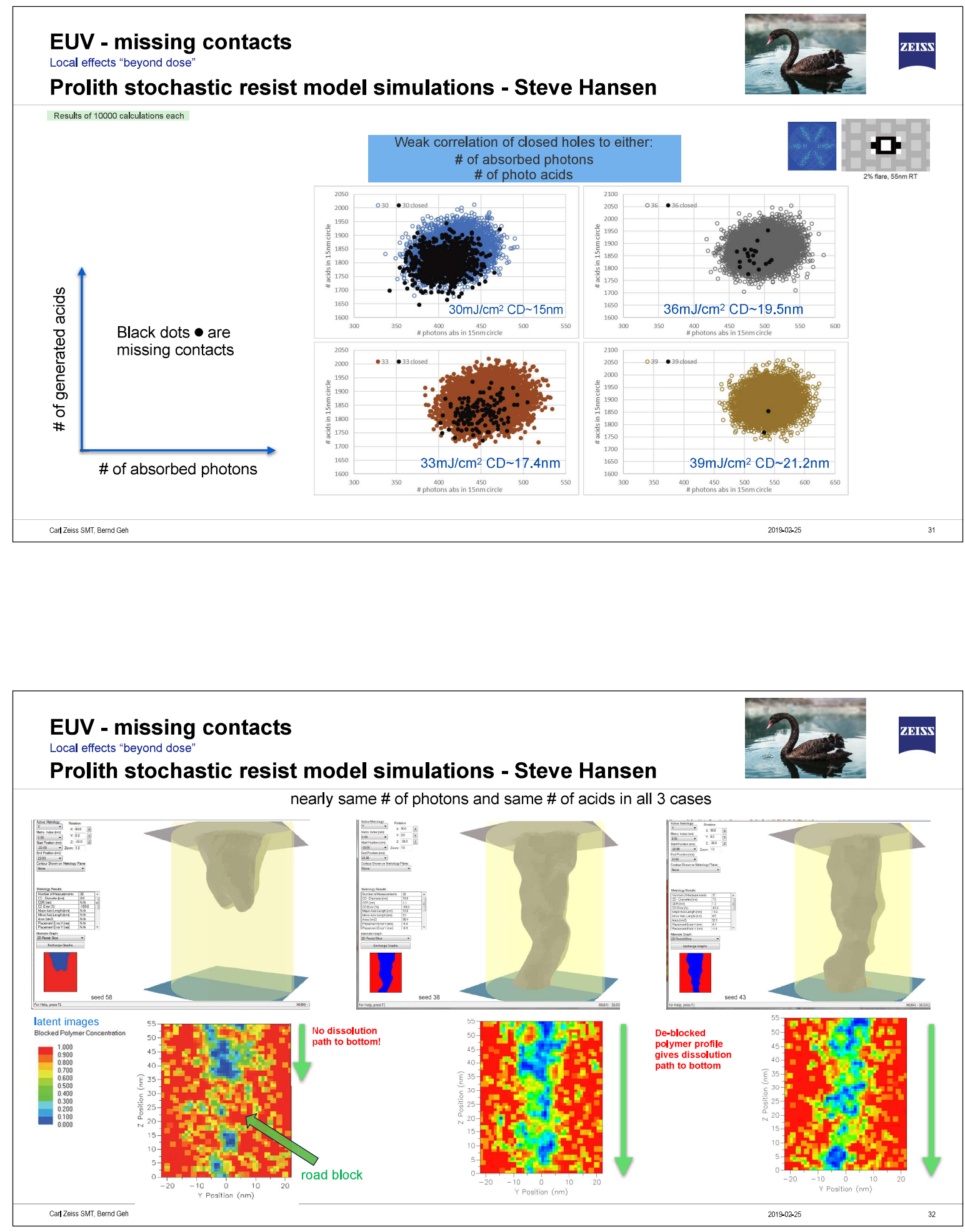

Proc. of SPIE Vol. 10957 1095705-16 


\section{Failing Contacts}

The relationship between LCDU and printing failures is very complex

Peter De Bisschop, "Stochastic effects in EUV lithography: random, local CD variability, and printing failures," JM 16(4), 041013 (2017)

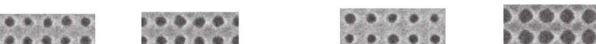

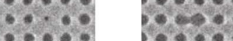

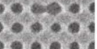

$\because: \cdots$

$\because \cdots$

$\ldots$

poes

poos
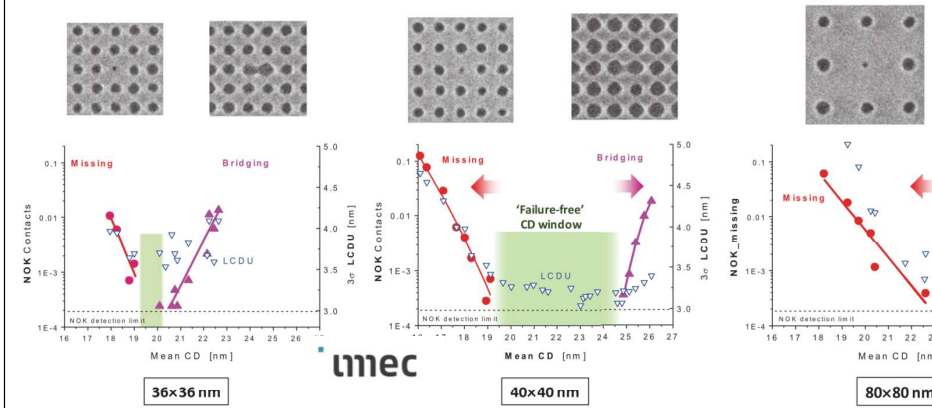

Don't miss:

Tuesday, 8:00am-8:20am: Stochastic printing failures in EUV lithography (Invited Paper from IMEC), [10957-10] Tuesday, 10:50am-11:10am: Impact of local variability on defect- aware process window degradation (Invited Paper), Mark John Maslow, et al, ASML, TEL, IMEC, [10957-13]

\section{Epilogue - a call to the next generation of bright engineers and scientists}

Always a good idea to listen to an old Lithographer! They've seen many things... 


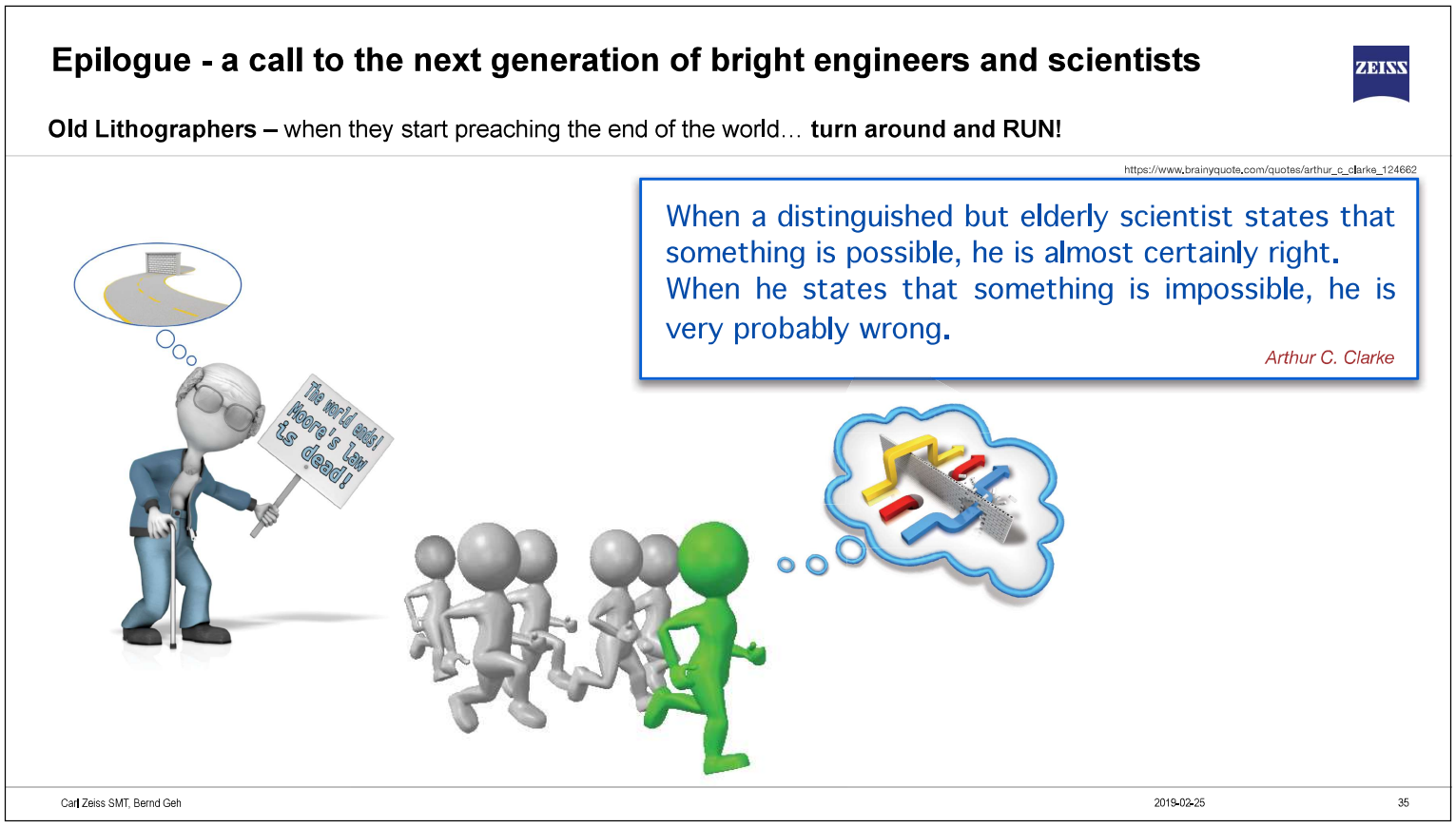

There's always new things to discover

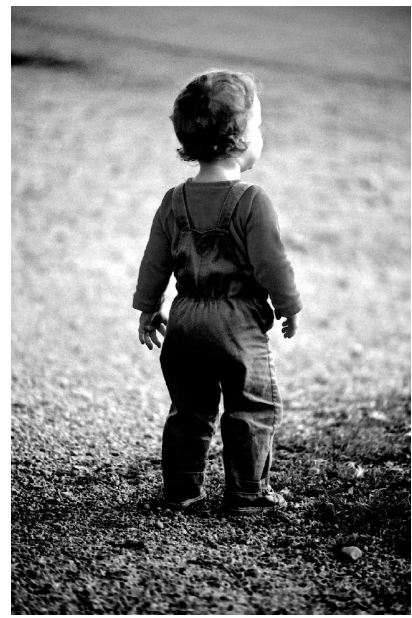

SPIE - Advanced Lithography 2019

Acknowledgements

Gijsbert Rispens

Steve Hansen

Extreme Ultraviolet (EUV) Lithography $\mathrm{X}$

Vladan Blahnik

Peter Graf

Jan van Schoot

Jo Finders

Rudi v. Bünau

Jörg Zimmermann

Peter de Bischop

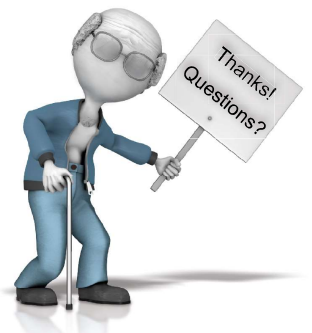

Peter de Bischop
Vicky Philipsen

Eelco van Setten

Renzo Capelli

Martin Dietzel

Ofir Sharoni

Klaus Edinger

Michael Waldow

Gregg Gallatin 